\title{
Towards Reliable Probabilistic Time-Series Projections of Global Mean Surface Temperature
}

\author{
Philip G. Sansom ( $\nabla$ p.g.sansom@exeter.ac.uk ) \\ University of Exeter School of Chemistry https://orcid.org/0000-0002-5596-4507 \\ Donald Cummins
}

University of Exeter

\section{Stefan Siegert}

University of Exeter

David B Stephenson

University of Exeter

\section{Research Article}

Keywords: Reliable Probabilistic Time-Series, Projections of Global Mean Surface, Temperature, global warming, pre-industrial conditions, probability

Posted Date: December 8th, 2021

DOI: https://doi.org/10.21203/rs.3.rs-1103739/v1

License: (c) (1) This work is licensed under a Creative Commons Attribution 4.0 International License. Read Full License 


\title{
Towards reliable probabilistic time-series projections of global
}

\author{
mean surface temperature
}

\author{
Philip G. Sansom, Donald Cummins, Stefan Siegert and David B. Stephenson
}

September 16, 2021

\begin{abstract}
Quantifying the risk of global warming exceeding critical targets such as $2.0^{\circ} \mathrm{C}$ requires reliable projections of uncertainty as well as best estimates of Global Mean Surface Temperature (GMST). However, uncertainty bands on GMST projections are often calculated heuristically and have several potential shortcomings. In particular, the uncertainty bands shown in IPCC plume projections of GMST are based on the distribution of GMST anomalies from climate model runs and so are strongly determined by model characteristics with little influence from observations of the realworld. Physically motivated time-series approaches are proposed based on fitting energy balance models (EBMs) to climate model outputs and observations in order to constrain future projections. It is shown that EBMs fitted to one forcing scenario will not produce reliable projections when different forcing scenarios are applied. The errors in the EBM projections can be interpreted as arising due to a discrepancy in the effective forcing felt by the model. A simple time-series approach to correcting the projections is proposed based on learning the evolution of the forcing discrepancy so that it can be projected into the future. This approach gives reliable projections of GMST when tested in a perfect model setting. When applied to observations this leads to projected warming of $2.2^{\circ} \mathrm{C}\left(1.7^{\circ} \mathrm{C}\right.$ to $\left.2.9^{\circ} \mathrm{C}\right)$ in 2100 compared to pre-industrial conditions, $0.4^{\circ} \mathrm{C}$ lower than a comparable IPCC anomaly estimate. The probability of staying below the critical $2.0^{\circ} \mathrm{C}$ warming target in 2100 more than doubles to 0.28 compared to only 0.11 from a comparably IPCC estimate.
\end{abstract}

\section{Introduction}

Global Mean Surface Temperature (GMST) is a key quantity for projecting future climate since it integrates many large scale processes, and many changes and impacts scale with GMST IPCC, 2018, Sutton et al. 2015]. It is also the summary measure most often used to communicate climate change to the public and to policy makers. Credible assessments of the risk of global warming exceeding targets such as $2{ }^{\circ} \mathrm{C}$ set out in the Paris agreement are critical for policy makers to make informed decisions about how to meet those targets. Therefore, it is important that the projections such assessments are based 
on are not only accurate, but also reliable, i.e., the probabilities of particular events are also accurate Broecker, 2012].

Climate projections are usually derived from general circulation models (GCMs) designed to simulate the climate system as closely as is currently possible Collins et al. 2013. Uncertainty in climate projections arises from many sources including imprecise initial conditions and natural variability Deser et al. 2012, the parameters of unresolved processes within a single GCM [Collins, 2007], choices made in constructing one GCM compared to another [Tebaldi and Knutti, 2007], and uncertainty about future emissions. Uncertainty about future emissions is usually addressed by conditioning projections on one or more predetermined scenarios Moss et al. 2010. Other uncertainties are usually quantified by analysing an ensemble of simulations that vary one or more of the uncertain factors.

The concept behind the use of ensembles for probabilistic projection is that the ensemble members represent samples from the distribution of plausible outcomes Smith, 2002, Palmer et al., 2006]. In practice, limitations of the models, observations etc. affect the spread of the ensemble and reduce the skill of the forecast. This is particularly problematic for multi-model ensembles which are not designed to span a space of possible model constructions, and are often referred to as "ensembles of opportunity" Knutti et al. 2010, Stephenson et al. 2012. Multi-model projections of GMST in particular exhibit a very large spread of outcomes [Collins, 2007]. The IPCC approach to handling this uncertainty is to take anomalies with respect to a specified reference period Collins, 2007, Figures T.S.14 \& T.S.15]. This reduces the spread of the projections in the future, but the projected warming and associated uncertainty then depend strongly on the reference period. There is also no reason to believe the projections are probabilistically reliable.

Many methods have been proposed for reducing the uncertainty in multi-model ensemble projections by weighting models according to their past performance in simulating the observed climate Greene et al., 2006, Min and Hense, 2006, Bhat et al., 2011, Shiogama et al., 2011; Watterson and Whetton, 2011]. Some weighting methods have been shown to produce reliable projections of future climate in perfect model tests Abramowitz and Bishop, 2015; Sanderson et al. 2017, Knutti et al., 2017, Strobach and $\mathrm{Bel}$, 2020. However, others have questioned the use of weights based on past performance when projecting conditions that differ significantly from that past Stainforth et al. 2007; Weigel et al., 2010. Sansom et al., 2013.

The alternative to model weighting is to build a formal statistical framework relating climate models to the real-world Räisänen and Palmer, 2001, Tebaldi et al., 2005, Furrer et al., 2007, Smith et al., 2009. Annan and Hargreaves, 2010, 2011. The advantage of a formal statistical framework is that the assumptions are transparent and more easily tested. The most recent developments proposed independently by Chandler 2013 and Rougier et al. 2013], and extended by Sansom et al. 2020] and Huang et al. 2020], allow for common biases between climate models and the real-world due to common limitations of the 


\section{${ }_{95} 2$ How reliable are energy balance models?}

Jonko et al. 2018 fitted two-box EBMs, however Fredriksen and Rypdal 2017 and Cummins et al. 2020 found that three-box EBMs provide a better fit to both models and observations. The three-box 


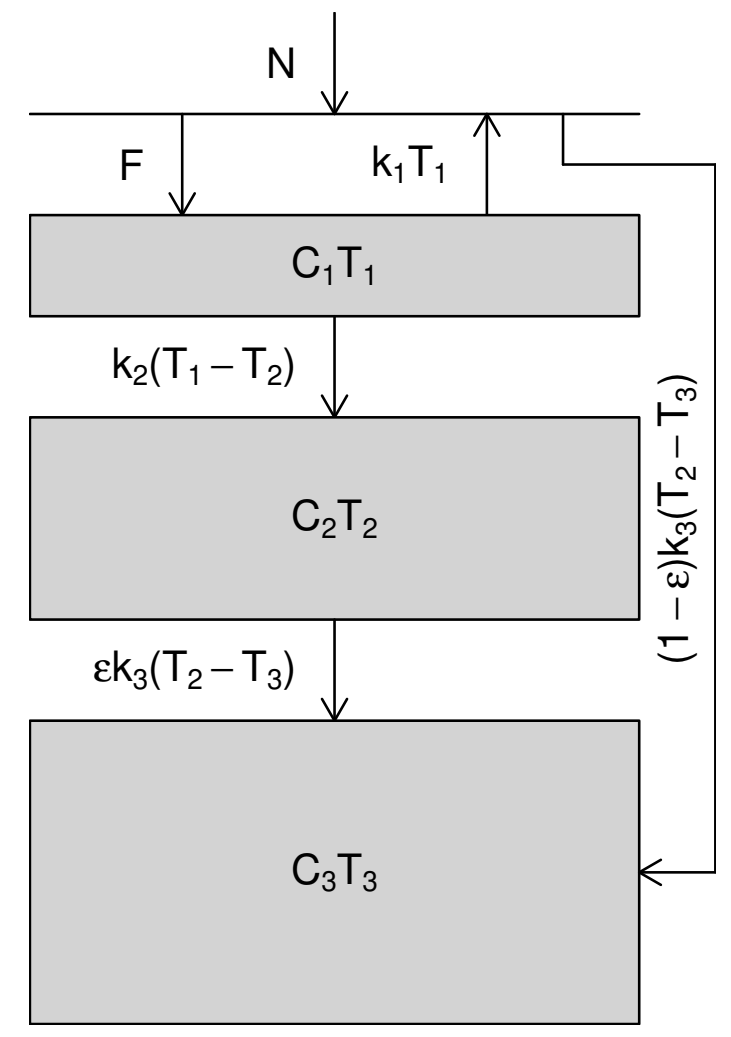

Figure 1: The 3-box EBM. The thickness of each box represents its heat capacity and the arrows indicate the flow of heat between boxes. The horizontal line represents the top of the atmosphere, which has no heat capacity.

model fitted by Cummins et al. 2020] is described by the following set of ordinary differential equations

$$
\begin{array}{ll}
C_{1} \frac{\mathrm{d} T_{1}}{\mathrm{~d} t}=F-k_{1} T_{1}-k_{2}\left(T_{1}-T_{2}\right)+w_{T}(t) \quad w_{T}(t) \sim \operatorname{Normal}\left(0, \sigma_{T}^{2}\right) \\
C_{2} \frac{\mathrm{d} T_{2}}{\mathrm{~d} t}=k_{2}\left(T_{1}-T_{2}\right)-\varepsilon k_{3}\left(T_{2}-T_{3}\right) \\
C_{3} \frac{\mathrm{d} T_{3}}{\mathrm{~d} t}=k_{3}\left(T_{2}-T_{3}\right)
\end{array}
$$

where $T_{1}, T_{2}$ and $T_{3}$ are the temperatures in each box, $C_{1}, C_{2}$ and $C_{3}$ are the heat capacities of the boxes, $k_{1}, k_{2}$ and $k_{3}$ are heat transfer coefficients, and $F$ represents external forcing (i.e., $\mathrm{CO}_{2}$ ). The top layer $T_{1}$ is usually assumed to represent the surface temperature and is the only observed quantity. The stochastic term $w_{T}(t)$ represents natural variability in surface temperature, and $\varepsilon$ is the so-called efficacy factor introduced by Held et al. 2010 to represent variation in $k_{1}$ during periods of transient warming. An EBM is a linear time-invariant system, so completely characterised by its step response. Therefore, EBMs are best fitted to idealised experiments containing a step change in forcing, such as the abrupt $4 \times \mathrm{CO}_{2}$ experiment used by Gregory et al. 2004]. However, energy balance models are overparameterised, making them difficult to fit even to step change experiments. This difficulty can be 
overcome by including measurements of the net downward radiation flux at the top of the atmosphere $N(t)$, in addition to surface temperature, to constrain $k_{1}$ via the following relation

$$
N(t)=F(t)-k_{1} T_{1}(t)+(1-\varepsilon) k_{3}\left[T_{2}(t)-T_{3}(t)\right]
$$

Note that measurements of $N(t)$ are only available when fitting to GCMs, not when fitting to the realworld. To allow the natural variability in $N(t)$ to differ from $T_{1}(t)$, Cummins et al. 2020] model the forcing $F(t)$ as red noise Hasselmann, 1976] so that

$$
\frac{\mathrm{d} F}{\mathrm{~d} t}=-\gamma\left[F-F_{C} X_{C}(t)\right]+w_{F}(t) \quad w_{F}(t) \sim \operatorname{Normal}\left(0, \sigma_{F}^{2}\right)
$$

where $F_{C}$ is the net radiative forcing due to a doubling of the atmospheric $\mathrm{CO}_{2}$ concentration and

$$
X_{C}(t)=\frac{1}{\log (2)} \log \left[\frac{\mathrm{CO}_{2}(t)}{\mathrm{CO}_{2}(0)}\right]
$$

96 where $\mathrm{CO}_{2}(t)$ is the $\mathrm{CO}_{2}$ concentration at time $t$ Geoffroy et al. 2013.

Although the EBM representation is defined in continuous time, we only have uniformly spaced discrete model outputs and observations of the surface temperature $T_{1}(t)$ and radiation balance $N(t)$. Cummins et al. 2020 showed that the EBM can be discretised and written in state-space form as

$$
\begin{aligned}
\boldsymbol{Y}(t) & =\boldsymbol{F}_{d} \boldsymbol{\theta}(t)+\boldsymbol{v}(t) & \boldsymbol{v}(t) & \sim \operatorname{Normal}\left(\mathbf{0}, \boldsymbol{V}_{d}\right) \\
\boldsymbol{\theta}(t) & =\boldsymbol{G}_{d} \boldsymbol{\theta}(t)+\boldsymbol{D}_{d} \boldsymbol{X}(t)+\boldsymbol{w}_{d}(t) & \boldsymbol{w}_{d}(t) & \sim \operatorname{Normal}\left(\mathbf{0}, \boldsymbol{W}_{d}\right)
\end{aligned}
$$

where $\boldsymbol{Y}(t)=\left[T_{1}(t), N(t)\right]^{\prime}$ for $t=1, \ldots, T$ are the data, $\boldsymbol{\theta}(t)=\left[F(t), T_{1}(t), T_{2}(t), T_{3}(t)\right]^{\prime}$ is the state, and $\boldsymbol{X}(t)$ is the forcing (see Appendix $\mathrm{A}$ for details). The stochastic term $\boldsymbol{v}(t)$ represents observation and measurement error which is set to zero for climate model output. The discretisation is exact provided the external forcing is piecewise constant, i.e., $X(t)$ is constant between times $t$ and $t+1$. Efficient maximum-likelihood estimation of the EBM parameters can be achieved by using the Kalman filter to evaluate the model likelihood (see Appendix A). Once the EBM parameters are estimated by fitting to abrupt $4 \times \mathrm{CO}_{2}$ experiments, it is straightforward to make predictions for other scenarios by applying appropriate $\mathrm{CO}_{2}$ forcing through $X_{C}(t)$. The maximum-likelihood fitting allows us to quantify not only projection uncertainty due to natural variability in forcing and temperature, but also uncertainty about the fitted parameters (see Appendix B for details).

Figure 2 shows the results of using the EBM fits to CMIP5 models by Cummins et al. 2020 to project future GMST and top-of-atmosphere radiation balance using the equivalent $\mathrm{CO}_{2}$ forcing for the CMIP5 historical and RCP4.5 scenarios Meinshausen et al. 2011. Figures 2(a) and (b) compare the EBM 
projections for the CanESM2 model with output from that model for the historical and RCP4.5 scenarios. CanESM2 is typical of the behaviour observed among the CMIP5 models analysed by Cummins et al. 2020]. The radiation balance looks reasonable, but there are clear biases in the GMST and the model output regularly exceeds the credible interval. Figures 2 (c) and (d) show the standardised prediction errors (see Appendix B] for all 16 climate models fitted by Cummins et al. [2020]. If the EBM projections were probabilistically reliable, then approximately $95 \%$ of the standardised errors should lie between -2 and 2 with good scatter between those bounds at all times (assuming the projections approximately follow a normal distribution). The radiation balance projections appear fairly reliable, with the exception of a series of strong negative spikes affecting all models during the historical period, and a slight positive bias visible in the multi-model mean error. However, the GMST projections are clearly not reliable, with standardised errors not only regularly but continuously exceeding six standard deviations, similar strong negative spikes during the historical period, and a positive mean bias that grows throughout the historical period.

The large standardised errors in Figures 2(c) and (d) indicate that the models are individually biased and over-confident, i.e. the credible intervals in Figures 2(a) and (b) are too narrow. The large multi-model mean bias indicates that there are common biases affecting all models. Neither the individual or common biases are surprising. The EBMs are very simple linear representations of a much more complicated non-linear system. Some of the biases may be due to the fact that the EBMs are forced with equivalent $\mathrm{CO}_{2}$ concentrations, whereas the GCMs are forced with individual greenhouse gas concentrations. However, there are many other processes and feedbacks included in the climate models (and the real-world) that are not represented in the EBMs, e.g., albedo changes due to sea and land ice loss. The effects of these processes will vary over time depending on the forcing scenario. Consequently, fitting an EBM to any single scenario will result in compensating errors in the parameters. Therefore, an EBM fitted to one scenario should not be expected to produce reliable projections when forced with a very different scenario.

One way to produce more reliable projections might be to fit to model outputs from abrupt $4 \times \mathrm{CO}_{2}$, historical and future scenarios simultaneously in order to find a common set of parameters. In practice, we found that this strategy results in parameters that do not fit any of the scenarios well. Figure 2 suggests a way forward. The spikes in both the radiation balance and GMST during the historical period in Figures 2(c) and (d) are due to volcanic eruptions injecting aerosols into the stratosphere, the effects of which are not included in RCP4.5 $\mathrm{CO}_{2}$ equivalent forcings, i.e., a missing component of forcing. Therefore, why not also treat the other biases as a discrepancy in effective forcing? Figure 2 and the EBM equations support this interpretation. There are large errors and a large bias in GMST, but almost none in the radiation balance. An effective discrepancy in forcing $F(t)$ directly affects both variables, but is balanced in the radiation balance equation by the negative $k_{1} T_{1}(t)$ term acting to cancel out the 
For the historical and future scenarios, we expand the EBM to include volcanic forcing $X_{V}(t)$ and a forcing discrepancy $\delta(t)$ so that

$$
\begin{aligned}
\frac{\mathrm{d} F}{\mathrm{~d} t} & =-\gamma\left[F-F_{C} X_{C}(t)-\boldsymbol{F}_{\boldsymbol{V}} \boldsymbol{X}_{\boldsymbol{V}}(\boldsymbol{t})\right]+w_{F}(t) \\
C_{1} \frac{\mathrm{d} T_{1}}{\mathrm{~d} t} & =F+\boldsymbol{\delta}-k_{1} T_{1}-k_{2}\left(T_{1}-T_{2}\right)+w_{T}(t) \\
C_{2} \frac{\mathrm{d} T_{2}}{\mathrm{~d} t} & =k_{2}\left(T_{1}-T_{2}\right)-\varepsilon k_{3}\left(T_{2}-T_{3}\right) \\
C_{3} \frac{\mathrm{d} T_{3}}{\mathrm{~d} t} & =k_{3}\left(T_{2}-T_{3}\right)
\end{aligned}
$$

where $F_{V}$ is the radiative coefficient of volcanic forcing, and

$$
N(t)=F(t)+\boldsymbol{\delta}(\boldsymbol{t})-k_{1} T_{1}(t)+(1-\varepsilon) k_{3}\left[T_{2}(t)-T_{3}(t)\right] .
$$

The additional terms are highlighted in bold. Equations $11-12$ are unchanged from Equations 2 3. but are included for completeness.

Figure 2 indicates that we need to split the discrepancy $\delta(t)$ into shared and model-specific compo- 
nents. If we try to model the shared and model-specific discrepancy components independently, then the statistical model is not identifiable since we have $M+1$ discrepancy components and only $M$ time-series, where $M$ is the number of models. Therefore, we model the forcing discrepancy as

$$
\frac{\mathrm{d} \delta}{\mathrm{d} t}=\nu(t)+w_{\delta}(t) \quad \nu(t) \sim \operatorname{Normal}\left(0, \sigma_{\nu}^{2}\right) \quad w_{\delta}(t) \sim \operatorname{Normal}\left(0, \sigma_{\delta}^{2}\right)
$$

where $w_{\delta}(t)$ is specific to particular climate model and $\nu(t)$ is common to all models. This parametrisation introduces two additional model-specific parameters $F_{V}$ and $\sigma_{\delta}$, and one shared parameter $\sigma_{\nu}$.

Equation 14 implies that, in discrete time, each model-specific discrepancy $\delta(t)$ is modelled by a random walk about a common mean which is itself modelled by a random walk. The "random-walkabout-a-random-walk" formulation is known variously as an integrated random walk Young et al. 1991, a polynomial growth model West and Harrison, 1997) or a local linear trend model Durbin and Koopman 2012, and has been widely used to model time varying trends. The formulation in Equation 14 is among the simplest that could address the biases seen in Figure 2 and imparts little prior information except a degree of smoothness controlled by the variances $\sigma_{\delta}^{2}$ and $\sigma_{\nu}^{2}$. In order to correct the biases in both model projections and the projections of the real-world, the shared discrepancy $\nu(t)$ itself, not just its variance $\sigma_{\nu}^{2}$ must be learned. Therefore, the formulation of $\nu(t)$ will have little effect on the projections provided it is sufficiently general to capture the underlying behaviour.

It is useful to define the common mean discrepancy $\alpha(t)$ as

$$
\frac{\mathrm{d} \alpha}{\mathrm{d} t}=\nu(t)
$$

Note that we refer to $\alpha(t)$ and $\nu(t)$ interchangeably as the shared discrepancy since although $\alpha(t)$ is the more interpretable quantity, it never actually enters the model formulation, so $\nu(t)$ is the object of inference.

\subsection{Learning the distribution over the models}

Each EBM representation has 13 model-specific parameters which we collect into a vector

$$
\phi_{m}=\left(\gamma, C_{1}, C_{2}, C_{3}, k_{1}, k_{2}, k_{3}, \varepsilon, \sigma_{F}, \sigma_{T}, F_{C}, F_{V}, \sigma_{\delta}\right)^{\prime}
$$

where subscript $m=1, \ldots, M$ denotes a specific climate model. We assume that the models are exchangeable, i.e., without prior knowledge about the performance of a particular climate model, we would specify the same prior beliefs about the parameters for every climate model in the ensemble. Therefore, 
it is convenient to model the relationship over the models as

$$
\log \left(\phi_{m}\right) \sim \operatorname{Normal}\left(\boldsymbol{\mu}_{\phi}, \boldsymbol{\Sigma}_{\phi}\right)
$$

for $m=1, \ldots, M$, where $\boldsymbol{\mu}_{\boldsymbol{\phi}}$ is a real vector of length 13 , and $\boldsymbol{\Sigma}_{\boldsymbol{\phi}}$ is a $13 \times 13$ symmetric positive-definite matrix. Specifying the distribution over the parameters on the log scale ensures that all of the parameters in $\phi_{m}$ are constrained to be positive.

In order to learn the individual model EBM representations $\phi_{m}(m=1, \ldots, M)$ and their distribution, parametrised by $\boldsymbol{\mu}_{\boldsymbol{\phi}}$ and $\boldsymbol{\Sigma}_{\boldsymbol{\phi}}$, we require simulations from abrupt $4 \times \mathrm{CO}_{2}$, historical and future experiments from each climate model, and the equivalent $\mathrm{CO}_{2}$ and stratospheric aerosol concentrations used to force those experiments.

\subsection{Learning about the real-world}

We assume that the real climate system can be approximated by an EBM identical to those used to represent the climate model outputs during the historical and future periods (Equations 9 14). The real climate system is assumed to have its own unique vector of parameters

$$
\phi_{Z}=\left(\gamma, C_{1}, C_{2}, C_{3}, k_{1}, k_{2}, k_{3}, \varepsilon, \sigma_{F}, \sigma_{T}, F_{C}, F_{V}, \sigma_{\delta}\right)^{\prime}
$$

where subscript $Z$ denotes the real-world. Following Rougier et al. [2013] and Sansom et al. [2020] we assume that the real climate system is co-exchangeable with the climate models such that

$$
\log \left(\phi_{Z}\right) \sim \operatorname{Normal}\left(\boldsymbol{\mu}_{\phi}, \kappa^{2} \boldsymbol{\Sigma}_{\boldsymbol{\phi}}\right)
$$

where $\kappa$ is a positive real scalar. This formulation implies that our expectation for the real-world is the same as the models, and the correlations between parameters are the same, but our uncertainty (reflected in the marginal variances) may be different due to missing processes and other errors in the models. Standard practice is to assume that climate models are exchangeable with the real-world, i.e, $\kappa=1$. The formulation in Equation 17 gives the flexibility to assess the impact of increased uncertainty due to missing processes etc. by setting $\kappa>1$.

In order to learn the EBM representation of the real-world, we require only historical observations of GMST in addition to the distribution of the EBM parametrised by $\boldsymbol{\mu}_{\boldsymbol{\phi}}$ and $\boldsymbol{\Sigma}_{\boldsymbol{\phi}}$ learned from the climate models. Ideally observations of the top-of-atmosphere radiation balance would also be used, but these are only available for a very limited period, so are omitted. The $\mathrm{CO}_{2}$ and stratospheric aerosol concentrations are assumed to be the same as in the historical model simulations. 
Our aim is to evaluate $\operatorname{Pr}\left(Z_{F} \mid X_{F}\right)$, the distribution of future climate $Z_{F}=\left\{Z\left(\tau_{H}+1\right), \ldots, Z\left(\tau_{F}\right)\right\}$ given future $\mathrm{CO}_{2}$ forcing $X_{F}=\left\{X_{C}\left(\tau_{H}+1\right), \ldots, X_{C}\left(\tau_{F}\right)\right\}$. In Section 3.4, we outlined how this can be done by Monte Carlo methods, sampling from

$$
\operatorname{Pr}\left(Z_{F} \mid X_{F}\right)=\int \operatorname{Pr}\left[Z_{F} \mid \phi_{Z}, \nu_{F}, \boldsymbol{\theta}_{Z}\left(\tau_{H}\right), X_{F}\right] \mathrm{d} \phi_{Z}, \nu_{F}, \boldsymbol{\theta}_{Z},
$$


where $\phi_{Z}$ are the parameters of the EBM representation of the real-world, $\nu_{F}=\left\{\nu\left(\tau_{H}+1\right), \ldots, \nu\left(\tau_{F}\right)\right\}$ is the shared discrepancy, and $\boldsymbol{\theta}_{Z}\left(\tau_{H}\right)=\left[F\left(\tau_{H}\right), T_{1}\left(\tau_{H}\right), T_{2}\left(\tau_{H}\right), T_{3}\left(\tau_{H}\right), \delta\left(\tau_{H}\right)\right]^{\prime}$ is the state of the realworld at the end of the historical/observed period.

However, we still need to be able to evaluate the posterior distribution of the EBM parameters $\phi_{Z}$ and the shared discrepancy $\nu$. This is achieved by Markov-Chain Monte Carlo sampling from the full posterior

$$
\operatorname{Pr}\left(\phi_{Z}, \nu \mid \mathcal{D}\right)=\int \operatorname{Pr}\left(\phi_{Z}, \nu, \phi_{1}, \ldots, \phi_{M}, \boldsymbol{\mu}_{\phi}, \boldsymbol{\Sigma}_{\boldsymbol{\phi}}, \sigma_{\nu} \mid Z_{H}, \kappa, \mathcal{D}\right) \mathrm{d} \phi_{1}, \ldots, \boldsymbol{\phi}_{M}, \boldsymbol{\mu}_{\boldsymbol{\phi}}, \boldsymbol{\Sigma}_{\boldsymbol{\phi}}, \sigma_{\nu}
$$

where $\mathcal{D}$ represents the available data, i.e., model outputs from the abrupt $4 \times \mathrm{CO}_{2}$, historical and future experiments, and $\mathrm{CO}_{2}$ and volcanic aerosol forcings for those experiments. In practice, we assume that

$$
\begin{aligned}
\operatorname{Pr}\left(\phi_{Z}, \nu, \phi_{1}, \ldots, \boldsymbol{\phi}_{M}, \boldsymbol{\mu}_{\phi}, \boldsymbol{\Sigma}_{\boldsymbol{\phi}}, \sigma_{\nu} \mid Z_{H}, \kappa, \mathcal{D}\right)= & \\
& \operatorname{Pr}\left(\phi_{Z} \mid \nu, \boldsymbol{\mu}_{\boldsymbol{\phi}}, \boldsymbol{\Sigma}_{\boldsymbol{\phi}}, \kappa, Z_{H}, X_{H}\right) \operatorname{Pr}\left(\nu, \boldsymbol{\phi}_{1}, \ldots, \boldsymbol{\phi}_{M}, \boldsymbol{\mu}_{\phi}, \boldsymbol{\Sigma}_{\boldsymbol{\phi}}, \sigma_{\nu} \mid \mathcal{D}\right) .
\end{aligned}
$$

This implies that the observations $Z_{H}$ do not contribute to the estimation of the shared discrepancy $\nu$ its variance $\sigma_{\nu}^{2}$, or the common parameters $\boldsymbol{\mu}_{\phi}$ and $\boldsymbol{\Sigma}_{\phi}$. This assumption is not necessary, but is stated for transparency, and intended to emphasise the role of the models in providing prior information for inference about the real-world. Full details of the priors for $\boldsymbol{\mu}_{\phi}, \boldsymbol{\Sigma}_{\boldsymbol{\phi}}$ and $\sigma_{\nu}$, and the partially collapsed Gibb's sampler used to sample the full posterior are given in the Supplementary Material. The process is simplified by conditioning on the shared discrepancy $\nu$, as we do when sampling the future climate in Section 3.4. Due to the inclusion of $\nu$, Equations $9 \sqrt{13}$ imply a $5 M$ dimensional state-space model for the joint distribution of the outputs from the climate models. The resulting joint likelihood function for $\phi_{1}, \ldots, \phi_{M}$ would be difficult and expensive to evaluate. Conditioning on $\nu$ simplifies the process by allowing us to fit $M$ independent 5 dimensional state-space models (see Appendix C) and evaluate $M$ much simpler likelihood functions instead, one for each climate model.

The abrupt $4 \times \mathrm{CO}_{2}$, historical and future runs all contribute to the likelihoods of the individual EBM representations $\boldsymbol{\phi}_{m}(m=1, \ldots, M)$, and hence the distribution of the EBM parameters $\left(\boldsymbol{\mu}_{\boldsymbol{\phi}}, \boldsymbol{\Sigma}_{\boldsymbol{\phi}}\right)$. However, most of the information comes from abrupt $4 \times \mathrm{CO}_{2}$ experiment with the stronger forcing. The historical and future runs primarily inform the coefficient of volcanic forcing $F_{V}$ and the standard deviations of the individual discrepancy $\sigma_{\delta}$ in each model, and the shared discrepancy $\nu(t)$ parametrised by $\sigma_{\nu}$. As stated above, the EBM representation of the real-world $\phi_{Z}$ is learned separately and depends only on the historical observations of the real world $Z_{H}$ and the distribution of the EBM parameters $\left(\boldsymbol{\mu}_{\boldsymbol{\phi}}, \boldsymbol{\Sigma}_{\boldsymbol{\phi}}\right)$ learned from the models. The same $\mathrm{CO}_{2}$ and volcanic forcings are assumed for the models and the real world during the historical and future periods. 
The results in Section 5 are based on samples from four parallel chains, initialised from well dispersed starting conditions (see Supplementary Material for details of initialisation). Each chain was run for a burn-in period of 25000 samples during which robust adaptive Metropolis-Hastings sampling Vihola, 2012 was used to learn optimal proposal distributions for $\phi_{1}, \ldots, \phi_{M}$ and $\sigma_{\nu}$. Each chain was then run for another 250000 samples with the proposal distributions fixed at their values at the end of the burn-in period. Gelman-Rubin diagnostics Gelman and Rubin, 1992 and visual inspection indicated that all variables converged successfully. The eight chains give a total of 2000000 samples of each variable, however for final storage only every 200th sample was kept. Therefore, the results in Section 5 are based on 10000 samples and 10000 corresponding future trajectories. All results are based on $\kappa=1.0$ in Equation 17] unless stated otherwise, i.e., the real-world is exchangeable with the models.

\subsection{Model checking}

In order to check whether the addition of the forcing discrepancy produces reliable estimates of future climate we perform a leave-one-out cross-validation. When trying to project the climate of the real-world, only observations of surface temperature for the historical period are available. Therefore, each climate model is excluded in turn, and only its output for surface temperature during the historical period is used in place of observations $Z_{H}$ of the real-world. The same MCMC procedure is then used to sample the posterior distribution of the parameters, but with only 4 chains and shorter burn-in and sampling periods of 25000 and 100000 samples respectively. For each excluded model, we retain every 40th sample to give 10000 samples in total, and project forward using the methodology described in Section 3.4 to give 10000 future trajectories. Standardised projection errors are computed as described in Appendix B

\subsection{Discussion}

When learning about the real-world, we only have around 170 years of historical observations to learn both the basic EBM parameters and the volcanic forcing and independent discrepancy parameters $F_{V}$ and $\sigma_{\delta}$. Therefore, using the models to estimate the prior for the parameters $\phi_{Z}$ in Equation 17 is critical for obtaining realistic inferences since the observations will provide only limited information. The fact that the shared discrepancy $\nu(t)$ is assumed to also apply to the real-world reflects the fact that $\nu(t)$ quantifies inadequacy in the ability of the EBM representations to approximate the more complete climate models. Any inadequacy in the climate models ability to approximate the real-world is accounted for by the inflation of the prior on the real-world EBM parameters $\phi_{Z}$ in Equation 17 .

The initial conditions for the EBM representations of the climate model outputs are well defined since the models are all initialised from a 500-year run under pre-industrial conditions after a lengthy spin-up which should ensure they are (almost) in equilibrium. However, for the real-world we have no observations for the pre-industrial period so we are forced to use the early industrial period 1850-1900 
as a reference. Also, we cannot be certain that the real system was in equilibrium prior to 1850 . The probabilistic representation used here means that the sensitivity of the projections to these assumptions can be explored through careful specification of the priors for the initial state, although we do not do so here.

While the projections will be insensitive to the formulation of the shared discrepancy $\nu(t)$, they will be more sensitive to the formulation of the model and real-world specific discrepancies $\delta(t)$. The random walk formulation implies that uncertainty about the state of the system will continue to increase even after the system has reached a new equilibrium. For very long range projections, e.g., several centuries, this behaviour is obviously undesirable. However, cross-validation indicates that the linear growth in uncertainty (variance) over time implied by this formulation is realistic for most current scenario climate change scenarios as far as the year 2100 (see Section 5.1 and Supplementary Material for details).

The assumption of exchangeability between the climate models in Section 3.2 implies that we would also specify the same prior beliefs about every pair, triple, etc. of models. In practical terms, this means that each model should be equally similar to every other model. For climate models this is clearly not the case. Some centres submit more than one model, or more than one version of the same model, some models from different centres share whole atmosphere or ocean component models. These models will be more similar than those that share no common components. To satisfy the assumption of exchangeability we analyse only a subset of the available models that we judge to be approximately exchangeable.

\section{Data}

In Section 5 we apply the methodology proposed in Section 3 to outputs from the CMIP5 multi-model ensemble. For each CMIP5 model we select run r1i1p1 from the pre-industrial control (piControl), abrupt $4 \times \mathrm{CO}_{2}$ (abrupt4xCO2), historical and RCP4.5 (rcp45) experiments. The variables used are near surface temperature (tas) and the top-atmosphere radiation balance (rsdt-rsut - rlut). Data are globally and annually averaged to give bivariate time-series of 500 years for the piControl experiment, 150 years for the abrupt $4 \times \mathrm{CO}_{2}$ experiment, and 251 years for the combined historical and RCP4.5 experiments. Some models have missing years at the end of the abrupt $4 \times \mathrm{CO}_{2}$ experiment or the beginning of the historical experiment. The Kalman filter methodology used to fit the EBMs (see Appendix A) can handle these missing values without special provision. In order to fit EBM representations to the model output we require temperature and radiation anomalies relative to an equilibrium state. Therefore, the mean of the piControl for each model is removed from the outputs of the abrupt $4 \times \mathrm{CO}_{2}$, historical and $\mathrm{RCP} 4.5$ experiments.

The assumption of exchangeability between models in Section 3.2 implies that every model should be equally similar to every other model. In order to satisfy this assumption, we analyse only a subset 
Table 1: CMIP5 models.

\begin{tabular}{lll}
\hline Centre & Model & Institution \\
\hline BCC & BCC-CSM1.1 & Beijing Climate Center, China \\
CCCma & CanESM2 & Canadian Centre for Climate Modelling and Analysis, Canada \\
NCAR & CCSM4 & National Center for Atmospheric Research (NCAR), United States \\
CNRM & CNRM-CM5 & Centre National de Recherches Mètèorologiques, France \\
LASG & FGOALS-s2 & Institute of Atmospheric Physics, China \\
GFDL & GFDL-ESM2G & Geophysical Fluid Dynamics Laboratory, United States \\
GISS & GISS-E2-R & NASA Goddard Institute for Space Studies, United States \\
MOHC & HadGEM2-ES & Met Office Hadley Centre, United Kingdom \\
IPSL & IPSL-CM5A-MR & Institut Pierre-Simon Laplace, France \\
MIROC & MIROC5 & Japan Agency for Marine-Earth Science and Technology, Japan \\
MPI-M & MPI-ESM-LR & Max Planck Institute for Meteorology, Germany \\
MRI & MRI-CGCM3 & Meteorological Research Institute, Japan \\
NCC & NorESM1-M & Norwegian Climate Centre, Norway \\
\hline
\end{tabular}

of the available models. The 13 models chosen are listed in Table 1. The subset is based on the thinned ensemble analysed by Sansom et al. [2020], where the models were chosen to minimise common components between models while maintaining similar horizontal and vertical resolutions. There are three differences compared to the ensemble analysed by Sansom et al. [2020]. The CCSM4 model based on the older CAM4 atmosphere models has been substituted for the more recent CESM1 model based on the updated CAM5 atmosphere, since not all the required runs were available from the CESM1 model. Similarly, the EC-EARTH model is missing due to a missing file in one of the required runs. Finally, the INM-CM4 model was excluded since it did not include volcanic forcing in the historical experiment. The chosen models are also similar to those analysed by Cummins et al. [2020] with the exception that we include GFDL-ESM2G and IPSL-CM5A-MR rather than GFDL-ESM2M and IPSL-CM5A-LR respectively.

For the real-world, we use the annual ensemble median GMST from the HadCRUT4 dataset Morice et al. 2012. The HadCRUT4 dataset provides anomalies relative to the 1961-1990 average, so the anomalies need to be adjusted for compatibility with the models. Since observations prior to 1850 are not readily available, we adopt the IPCC SR1.5 approach and re-reference the anomalies to the 18501900 average $I P C C, 2018$. HadCRUT4 also includes an extensive quantification of the uncertainties associated with the observations. We use the provided lower and upper $95 \%$ confidence bounds for the combined effects of all of the uncertainties on the annual time-series to compute the annual standard deviation of the observation uncertainty assuming the observations follow a normal distribution. We use these standard deviations as our estimate of the independent annual observation uncertainty. This is likely to be an over-estimate of the implied uncertainty since the true uncertainty is likely to be correlated between years. Although observations of top-of-atmosphere radiation do exist, they are limited to the satellite era (1970s onwards) making them difficult to use in this context, so we choose not to include them.

To drive the EBM representations we use the $\mathrm{CO}_{2}$ equivalence concentrations from the CMIP5 
Table 2: Parameter estimates. The posterior means of the parameters EBM representations of the CMIP5 models, the CMIP5 ensemble and the observed climate.

\begin{tabular}{lccccccccccccc}
\hline Model & $\gamma$ & $C_{1}$ & $C_{2}$ & $C_{3}$ & $k_{1}$ & $k_{2}$ & $k_{3}$ & $\varepsilon$ & $\sigma_{F}$ & $\sigma_{T}$ & $F_{C}$ & $F_{V}$ & $\sigma_{\delta}$ \\
\hline BCC-CSM1.1 & 3.50 & 3.93 & 9.4 & 53 & 1.23 & 2.71 & 0.69 & 1.28 & 0.64 & 0.37 & 3.60 & 23.8 & 0.048 \\
CanESM2 & 2.03 & 4.02 & 11.2 & 71 & 0.98 & 2.12 & 0.74 & 1.32 & 0.61 & 0.54 & 3.94 & 18.6 & 0.035 \\
CCSM4 & 2.52 & 4.40 & 13.4 & 78 & 1.29 & 2.03 & 1.22 & 1.37 & 0.62 & 0.51 & 4.06 & 23.1 & 0.039 \\
CNRM-CM5 & 3.54 & 3.55 & 10.2 & 82 & 1.14 & 2.66 & 0.64 & 0.97 & 0.56 & 0.44 & 3.66 & 21.0 & 0.048 \\
FGOALS-s2 & 2.05 & 4.56 & 11.5 & 134 & 0.83 & 1.67 & 1.17 & 1.28 & 0.72 & 0.64 & 3.90 & 20.0 & 0.034 \\
GFDL-ESM2G & 2.36 & 4.74 & 14.8 & 104 & 1.46 & 1.79 & 1.48 & 1.31 & 0.71 & 0.54 & 3.63 & 23.1 & 0.038 \\
GISS-E2-R & 2.80 & 5.09 & 29.0 & 118 & 1.78 & 1.93 & 3.31 & 1.44 & 0.45 & 0.35 & 4.11 & 23.4 & 0.037 \\
HadGEM2-ES & 1.96 & 4.13 & 10.0 & 91 & 0.62 & 2.34 & 0.66 & 1.37 & 0.60 & 0.40 & 3.20 & 14.8 & 0.031 \\
IPSL-CM5A-MR & 2.27 & 3.90 & 12.0 & 95 & 0.79 & 2.45 & 0.75 & 1.19 & 0.52 & 0.41 & 3.47 & 16.1 & 0.034 \\
MIROC5 & 1.58 & 4.43 & 22.6 & 130 & 1.61 & 1.55 & 1.77 & 1.17 & 0.52 & 0.80 & 4.45 & 19.3 & 0.032 \\
MPI-ESM-LR & 2.05 & 4.08 & 13.1 & 74 & 1.13 & 1.98 & 0.95 & 1.33 & 0.57 & 0.61 & 4.38 & 20.3 & 0.036 \\
MRI-CGCM3 & 2.18 & 3.96 & 13.5 & 66 & 1.20 & 2.62 & 0.74 & 1.27 & 0.54 & 0.39 & 3.32 & 16.6 & 0.036 \\
NorESM1-M & 1.85 & 4.74 & 17.2 & 107 & 1.11 & 2.05 & 1.44 & 1.42 & 0.57 & 0.44 & 3.45 & 16.7 & 0.030 \\
\hline Ensemble & 2.37 & 4.29 & 14.5 & 94 & 1.18 & 2.16 & 1.21 & 1.29 & 0.59 & 0.50 & 3.78 & 19.8 & 0.037 \\
\hline Observations & 2.07 & 4.39 & 17.0 & 102 & 1.17 & 2.24 & 1.32 & 1.34 & 0.54 & 0.45 & 3.59 & 17.2 & 0.033 \\
\hline
\end{tabular}

340

concentrations datasets [Meinshausen et al., 2011]. Although most major forcings were specified for the

CMIP5 experiments, stratospheric injection of sulfate aerosols from explosive volcanic eruptions was not Driscoll et al. 2012. However, most modelling groups chose to impose the stratospheric emissions from volcanic eruptions and the effects are clearly visible in Figure 2, To account for stratospheric aerosol emissions, we use the updated global mean stratospheric aerosol optical depth at $550 \mathrm{~nm}$ dataset by Sato et al. 1993 available from NASA GISS (https://data.giss.nasa.gov/modelforce/strataer/).

\section{Results}

The posterior means of the EBM parameters for each climate model, and those for the real-world are shown in Table 2, As outlined in Section 3, all parameters are estimated simultaneously rather than one model at a time as in Jonko et al. 2018 and Cummins et al. 2020. Overall, the posterior mean estimates of the main EBM parameters (excluding $F_{V}$ and $\sigma_{\delta}$ ) are very similar to those of Cummins et al. 2020]. This emphasises that most of the information about the EBM parameters still comes from the abrupt $4 \times \mathrm{CO}_{2}$ experiment. There were two unusual parameter estimates reported by Cummins et al. 2020], CNRM-CM5.1 had an unusually large value for $\gamma$ relative to the other models, and GISS-E2-R reported a very high value for $k_{3}$. The regularisation imposed by the common prior over the models in Equation 16 has constrained both of these parameters to values more similar to the other models, although the value of $k_{3}$ for GISS-E2-R is still almost double the next closest model.

\section{$5.1 \quad$ Model checking}

The posterior distribution of the shared discrepancy $\alpha(t)$ is plotted in Figure 3 and roughly follows the pattern seen in Figure 2, peaking around the year 2000 before slowly declining. The 10000 future 
trajectories from each model sample the posterior predictive distribution for that model. An example of the posterior predictive distributions is shown for CanESM2 in Figure 4(a) and (b). The temperature projections are biased low, but the credible interval now includes most of the model output, and the radiation balance is still well predicted.

To check the overall reliability of the projections we plot the standardised predictive error of each model from the cross-validation at times $t=2020, \ldots, 2100$ in Figure 4 (c) and (d). If the projections are reliable, then approximately $95 \%$ of the standardised errors should lie between -2 and 2 standard deviations at all times. For surface temperature, we see this is approximately the case. The projections appear very reliable, with good scatter between -2 and 2 standard errors and almost no overall mean bias. This contrasts sharply with Figure 2(c), indicating much improved projections. For the radiation balance in Figure 4(d), the projections also appear reliable, with little or no mean bias.

The cross-validation gives us confidence that even by assimilating only observations of temperature for the real-world we can obtain reliable projections of future surface temperature. The mean bias has been almost completely removed and the projections now appear reliable or slightly under-confident rather than very over-confident as in Figure 2 .

The Supplementary Material also includes extensive additional analysis checking the sensitivity of our inferences to our choice of priors, choice of climate models and choice of the coexchangeable coefficient $\kappa$. Our inferences are insensitive to the choice of priors and surprisingly insensitive to the coexchangeable coefficient $\kappa$. Inferences are not strongly influenced by the choice of climate models, but the sensitivity analysis does highlight the potential for bias due to including multiple variants of the same model.

\subsection{Equilibrium Climate Sensitivity}

The posterior mean estimates of the parameters of the real-world in Table 2 differ very little from those of the ensemble given by $\boldsymbol{\psi}$ with the exception of the heat transfer coefficients $k_{1}, k_{2}$ and $k_{3}$. Figure 5 shows the posterior distribution of the ECS of the real-world, given by $F_{C} / k_{1}$. The posterior distribution of ECS for the CMIP5 ensemble is also shown, estimated by sampling new values of $F_{C}$ and $k_{1}$ from Equation 16 conditional on the posterior samples of $\boldsymbol{\psi}$. Due to the limited signal in the historical temperature observations, there is insufficient information to usefully constrain the ECS of the real-world compared to the CMIP5 ensemble. For the real-world, we estimate a median ECS of $3.2^{\circ} \mathrm{C}$ and $90 \%$ credible interval $2.1^{\circ} \mathrm{C}$ to $5.1^{\circ} \mathrm{C}$. For the CMIP5 ensemble we estimate a median ECS of $3.3^{\circ} \mathrm{C}$ and $90 \%$ credible interval $2.1^{\circ} \mathrm{C}$ to $5.3^{\circ} \mathrm{C}$.

Various authors have tried to constrain estimates of ECS using a variety of metrics, see Brient 2020] or Hall et al. 2019 and references therein for examples, although the credibility of some of these estimates has been questioned Caldwell et al. 2018. Table 3 compares our estimate with that of several recent studies, including the synthesis report by Sherwood et al. 2020. Compared to the previous Bayesian 
Table 3: Estimates of Equilibrium Climate Sensitivity from the CMIP5 ensemble. Note that the IPCC interval is the "likely" $66 \%$ interval, not a $90 \%$ interval.

\begin{tabular}{|c|c|c|}
\hline Study & Median & $5 \%$ to $95 \%$ \\
\hline IPCC & & $1.5^{\circ} \mathrm{C}$ to $4.5^{\circ} \mathrm{C}$ \\
\hline Cox et al. Cox et al. 2018 & $2.8^{\circ} \mathrm{C}$ & $1.6^{\circ} \mathrm{C}$ to $4.0^{\circ} \mathrm{C}$ \\
\hline Jonko et al. Jonko et al. 2018 . & $2.5^{\circ} \mathrm{C}$ & $1.2^{\circ} \mathrm{C}$ to $3.9^{\circ} \mathrm{C}$ \\
\hline Jiménez-de-la-Cuesta \& Mauritsen Jiménez-de-la Cuesta and Mauritsen [2019] & $2.8^{\circ} \mathrm{C}$ & $1.7^{\circ} \mathrm{C}$ to $4.1^{\circ} \mathrm{C}$ \\
\hline Nijsse et al. Nijsse et al. 2020 & $2.3^{\circ} \mathrm{C}$ & $1.0^{\circ} \mathrm{C}$ to $4.1^{\circ} \mathrm{C}$ \\
\hline Sherwood et al. Sherwood et al. 2020 & $3.1^{\circ} \mathrm{C}$ & $2.3^{\circ} \mathrm{C}$ to $4.7^{\circ} \mathrm{C}$ \\
\hline This study & $3.2^{\circ} \mathrm{C}$ & $2.1^{\circ} \mathrm{C}$ to $5.1^{\circ} \mathrm{C}$ \\
\hline
\end{tabular}

Table 4: Estimates of future warming under RCP4.5 scenario averaged over 2081-2100. Note that the IPCC and Sherwood et al. Sherwood et al. [2020] intervals are $66 \%$ intervals, not $90 \%$ intervals. Note also, future warming cannot be compared to Figure 6 due to the different reference period.

\begin{tabular}{l|lllll}
\hline Study & Ensemble & Reference & Median & $5 \%$ to $95 \%$ \\
\hline IPCC & & CMIP5 & $1986-2005$ & $1.8^{\circ} \mathrm{C}$ & $1.1^{\circ} \mathrm{C}$ to $2.6^{\circ} \mathrm{C}$ \\
Sherwood et al. Sherwood et al. & 2020 & CMIP5 & $1986-2005$ & $1.8^{\circ} \mathrm{C}$ & $1.4^{\circ} \mathrm{C}$ to $2.3^{\circ} \mathrm{C}$ \\
Strobach and Bel Strobach and Bel & 2020 & CMIP5 & $1986-2005$ & $1.8^{\circ} \mathrm{C}$ & $1.7^{\circ} \mathrm{C}$ to $2.1^{\circ} \mathrm{C}$ \\
Tokarska et al. Tokarska et al. & 2020 & CMIP6 & $1995-2014$ & $1.8^{\circ} \mathrm{C}$ & $1.2^{\circ} \mathrm{C}$ to $2.5^{\circ} \mathrm{C}$ \\
This study & & CMIP5 & $1986-2005$ & $1.5^{\circ} \mathrm{C}$ & $1.1^{\circ} \mathrm{C}$ to $2.1^{\circ} \mathrm{C}$ \\
\hline
\end{tabular}

hierarchical analysis by Jonko et al. [2018], our median estimate is higher, although our credible interval similar in width. This study was not targeted specifically at constraining ECS, so it is not surprising that other studies have proposed estimates that differ more strongly from the median of the models. However, both our median estimate and credible interval are very similar to those of the synthesis report by Sherwood et al. 2020].

\subsection{Future projections}

Figure 6 shows the projections for the real-world under the RCP4.5 scenario based on the EBM fit to historical observations and accounting for shared and unique forcing bias. The projections make the usual assumption that the real-world is exchangeable with the climate model ensemble, i.e., $\kappa=1.0$. The projections are well constrained and lie entirely in the lower half of the range predicted by the CMIP5 ensemble. The mean surface temperature increase above pre-industrial conditions projected in 2100 is $2.2^{\circ} \mathrm{C}$ with $90 \%$ credible interval $1.7^{\circ} \mathrm{C}$ to $2.9^{\circ} \mathrm{C}$. This compares with an enlarged CMIP5 ensemble (sampling new models from Equation 16) with mean $2.5^{\circ} \mathrm{C}$ with $90 \%$ credible interval $1.6^{\circ} \mathrm{C}$ to $4.0^{\circ} \mathrm{C}$ in 2100 , and an IPCC-method estimate of $2.6^{\circ} \mathrm{C}$ with $90 \%$ credible interval $1.8^{\circ} \mathrm{C}$ to $3.4{ }^{\circ} \mathrm{C}$. These projections include natural variability, but not uncertainty about future observations.

Table 4 compares our projections of the mean warming in the period 2081-2100 above the 1986-2005 average with other recent studies. Our estimated median warming of $1.5^{\circ} \mathrm{C}$ is $0.3^{\circ} \mathrm{C}$ lower than the other recent estimates which all agree on warming of around $1.8^{\circ} \mathrm{C}$ by the end of the century. Our credible interval is also narrower than those of the IPCC or Tokarska et al. [2020], similar to the synthesis estimate of Sherwood et al. 2020], but wider than that of Strobach and Bel 2020]. 
Jonko et al. 2018 only made projections under the stronger RCP8.5 scenario. Our full analysis of the RCP8.5 scenario is included in the Supplementary Material. Under the RCP8.5 forcing scenario, Jonko et al. 2018 estimate a $90 \%$ credible interval of $2.2^{\circ} \mathrm{C}$ to $5.6^{\circ} \mathrm{C}$ in the year 2100 compared to the preindustrial period (no median estimate was given). In comparison, we estimate a median warming of $4.3^{\circ} \mathrm{C}$ with $90 \%$ credible interval $3.4^{\circ} \mathrm{C}$ to $5.6{ }^{\circ} \mathrm{C}$. Given the positive skewness in the majority of estimates, our median warming is likely higher than that of Jonko et al. [2018], but our credible interval is much narrower, despite the methodological similarity. The difference in the median can be explained by our inclusion of the shared forcing discrepancy, correcting for the tendency of the EBMs to underestimate the warming in Figure 2, The difference in credible interval is likely due to the fact that Jonko et al. 2018] estimate the distribution over the models in Equation 16 from the distribution of the individual EBM parameter estimates, whereas we learn both the individual and ensemble parameters simultaneously.

This results in some regularisation (shrinkage) of the individual estimates towards the consensus of the ensemble, and so a tighter distribution over the models. Since the model distribution acts as a prior for the real-world, this in turn results in a tighter distribution for the real-world.

Figure 7 shows the probabilities of meeting the targets set out in the Paris Agreement under the RCP4.5 scenario. Under the constrained projections, there is almost no probability of GMST exceeding $2.0^{\circ} \mathrm{C}$ in a particular year before 2040 , including fluctuations due to natural variability. After 2040 the probability rises rapidly at first then more slowly until it reaches 0.72 in 2100 in Figure 7(a). This is in striking contrast to the IPCC anomaly method which gives a non-zero probability as early as 2020 and a probability of $0.89 \mathrm{in} 2100$. The outlook for remaining below $1.5^{\circ} \mathrm{C}$ is less optimistic. The constrained projections agree with the anomaly projections that under the RCP4.5 scenario there is a probability of 0.99 that GMST will exceed $1.5^{\circ} \mathrm{C}$ above pre-industrial levels in 2100 .

\section{Conclusions}

In this study we propose a physically motivated statistical approach to producing bias-corrected timeseries projections of future GMST. Our approach builds on existing methods for combining projections from multiple climate models with observations of the real-world by incorporating physically motivated statistical representations of both climate model outputs and observations. Our method produces whole time-series of projections, enabling more detailed insights than time-slice averages. Biases in future projections are greatly reduced by the inclusion of a discrepancy in the effective forcing due to processes not captured by the simplified representation but whose effects may vary between forcing scenarios leading to biased projections. The proposed methodology not only provides point projections at each future time, but associated credible intervals and probabilities, accounting for natural variability, observation uncertainty, structural uncertainty and model inadequacy. 
Our projections indicate substantially less warming by the end of the 21st century than previous analyses. Our mean projection for GMST rise in 2100 compared to the pre-industrial period is $2.2^{\circ} \mathrm{C}$ $\left(1.7^{\circ} \mathrm{C}\right.$ to $\left.2.9^{\circ} \mathrm{C}\right)$, compared to the IPCC estimate of $2.6^{\circ} \mathrm{C}\left(1.8^{\circ} \mathrm{C}\right.$ to $\left.3.4^{\circ} \mathrm{C}\right)$. Several recent analyses project warming of around $1.8^{\circ} \mathrm{C}$ by the end of the 21 st century, compared to the end of the 20th century. In contrast we project only $1.5^{\circ} \mathrm{C}\left(1.1^{\circ} \mathrm{C}\right.$ to $\left.2.1^{\circ} \mathrm{C}\right)$. Perhaps the most encouraging result, is that the probability of exceeding $2.0^{\circ} \mathrm{C}$ by the end of the 21 st century is reduced from 0.89 using the IPCC anomaly method to 0.72 using our bias corrected method.

Compared to existing heuristic anomaly methods, observations of the real-world are integral to our projections, rather than simply an offset applied to an ensemble of models. Observations of the historical period contain limited information about the EBM representation. However, we have shown that by combining a suitable prior based on climate model outputs, there is enough information to usefully constrain projections of future climate. Rather than anomalies from an arbitrary reference period, we propose a physically motivated and bias-corrected times-series model which we have shown to be approximately reliable until the end of the 21st century. The statistical model makes the assumptions about the relationship between the models and the real world transparent. Our method has particular advantages for estimating sensitive quantities like quantiles or probabilities where the precision is limited by the number of climate models when using anomaly methods. Using our proposed time-series method, even these sensitive quantities can be estimated to any desired precision (up to Monte Carlo error) by drawing more plausible trajectories for the future climate.

In order to avoid biasing our projections towards models or components that are over-represented in the CMIP5 ensemble, we chose to infer our prior for the real-world based on only a subset of the available climate models. In doing so, we risk losing valuable information contained in the excluded models. However, comparing projections from the subset against projections using the full ensemble suggests that any information loss is very limited.

The choice to base our projections on the CMIP5 ensemble rather than the more recent CMIP6 ensemble was deliberate. The CMIP6 ensemble is well known to contain a number of models with very high ECS Meehl et al., 2020]. While there is some agreement about the cause of the high sensitivity, the plausibility of these models has yet to be determined. In addition, including any of the models with very high sensitivity would violate the assumption of exchangeability between models. Therefore, we choose to analyse the earlier CMIP5 ensemble instead.

Our results demonstrate that by working with physically interpretable representations and parameters, it is possible to obtain strongly constrained projections without the need to adopt performance based model weights. Having methods available that make very different assumptions is a healthy thing, since those assumptions can then be challenged and tested. The strength of the model weighting approach is that all available models can be included in the analysis. Further research is required to combine 
prior knowledge with dependences diagnosed from model outputs to enable the inclusion of all available models within formal statistical frameworks.

In forming our projections, we only used one run of each scenario from each model, when several runs of some scenarios are available from some models. By not using all available runs we are potentially throwing away valuable information. The methodology proposed here could easily be expanded to include multiple initial conditions runs without biasing our inferences towards the models with the most runs. However, the EBM representation arguably makes the inclusion of multiple runs of less value than might otherwise be the case. The EBM representation is a linear dynamic system. Therefore the response to a linear combination of inputs, i.e., known forcing and natural variability, is equal to the sum of the responses of the individual inputs. So by learning the parametric representation of the forced response, we are simultaneously learning the response to natural variability and vice-versa.

In principle, the methodology proposed here could be modified to project any future $\mathrm{CO}_{2}$ emissions scenario. Due to the presence of shared biases in the effective forcing, we chose to learn a shared component of forcing from simulations of the future as well as historical period. This makes our parameter inference and projections specific to a particular emissions scenario and limits us to making projections of scenarios for which we have model outputs to learn from. However, if the shared discrepancy and model-specific discrepancy parameters were only learned from the historical period, the simulations could then be allowed to evolve freely following any future emissions scenario. The resulting projections would have greater uncertainty than those shown here in order to account for future changes in the shared component which is then treated as unknown. However, both the shared and specific discrepancies would require more careful specification to ensure that projections are both credible and reliable. We have shown that reliable and well constrained century scale projections are possible using simulations from only a few climate models, without having to make these additional assumptions.

\section{Acknowledgement}

The authors would like to thank Mark Williamson for helpful discussions and comment. In addition, we acknowledge the World Climate Research Programme's Working Group on Coupled Modelling, which is responsible for CMIP, and we thank the climate modeling groups (listed in Table 1 of this paper) for producing and making available their model output. For CMIP the U.S. Department of Energy's Program for Climate Model Diagnosis and Intercomparison provides coordinating support and led development of software infrastructure in partnership with the Global Organization for Earth System Science Portals. 


\section{A State-space representation and discretisation}

Equations 15 can be written as

$$
\frac{\mathrm{d} \boldsymbol{\theta}}{\mathrm{d} t}=\boldsymbol{G} \boldsymbol{\theta}(t)+\boldsymbol{D} \boldsymbol{X}(t)+\boldsymbol{w}(t) \quad \boldsymbol{w}(t) \sim \operatorname{Normal}(\mathbf{0}, \boldsymbol{W})
$$

where

$$
\boldsymbol{D}=\left[\begin{array}{c}
\gamma F_{C} \\
0 \\
0
\end{array}\right] \quad \boldsymbol{X}(t)=\left[X_{C}(t)\right]
$$

and

$$
\boldsymbol{G}=\left[\begin{array}{cccc}
-\gamma & 0 & 0 & 0 \\
\frac{1}{C_{1}} & -\frac{k_{1}+k_{2}}{C_{1}} & \frac{k_{2}}{C_{1}} & 0 \\
0 & \frac{k_{2}}{C_{2}} & -\frac{k_{2}+\varepsilon k_{3}}{C_{2}} & \frac{\varepsilon k_{3}}{C_{2}} \\
0 & 0 & \frac{k_{3}}{C_{3}} & -\frac{k_{3}}{C_{3}}
\end{array}\right] \quad \boldsymbol{W}=\left[\begin{array}{cccc}
\sigma_{F}^{2} & 0 & 0 & 0 \\
0 & \frac{\sigma_{T}^{2}}{C_{1}^{2}} & 0 & 0 \\
0 & 0 & 0 & 0 \\
0 & 0 & 0 & 0
\end{array}\right]
$$

Following Cummins et al. 2020, the discretised state-space form of the EBM in Equations 7 and 8 is

$$
\boldsymbol{F}_{d}=\left[\begin{array}{cccc}
0 & 1 & 0 & 0 \\
1 & -k_{1} & (1-\varepsilon) k_{3} & -(1-\varepsilon) k_{3}
\end{array}\right] \quad \quad \boldsymbol{V}_{d}=\left[\begin{array}{ll}
0 & 0 \\
0 & 0
\end{array}\right]
$$

where

$$
\boldsymbol{G}_{d}=e^{\boldsymbol{G}} \quad \boldsymbol{D}_{d}=\boldsymbol{G}^{-1}\left(\boldsymbol{G}_{d}-\boldsymbol{I}\right) \boldsymbol{D} \quad \boldsymbol{W}_{d}=\int_{s=0}^{1} e^{\boldsymbol{G} s} \boldsymbol{W} e^{\boldsymbol{G}^{\prime} s}
$$

514 are the discretised forms of the matrices $\boldsymbol{G}, \boldsymbol{D}$ and $\boldsymbol{W}$.

\section{Model likelihood}

516 For parameter fitting by maximum likelihood in discrete time, the EBM likelihood factorises as

$$
\operatorname{Pr}[\boldsymbol{Y}(1), \ldots, \boldsymbol{Y}(T) \mid \boldsymbol{X}(1), \ldots, \boldsymbol{X}(T), \boldsymbol{\phi}]=\prod_{t=1}^{T} \operatorname{Pr}[\boldsymbol{Y}(t) \mid \mathcal{D}(t-1), \boldsymbol{\phi}]
$$




$$
[\boldsymbol{Y}(t) \mid \mathcal{D}(t-1), \boldsymbol{\phi}] \sim \operatorname{Normal}[\boldsymbol{f}(t), \boldsymbol{Q}(t)]
$$

where

$$
\boldsymbol{f}(t)=\boldsymbol{F}_{d} \boldsymbol{a}(t)
$$

\section{${ }_{530}$ Update step}

${ }_{531}$ The posterior distribution of the state $\boldsymbol{\theta}$ at time $t$ given the data at time $t$ is then

$$
[\boldsymbol{\theta}(t) \mid \mathcal{D}(t), \boldsymbol{\phi}] \sim \operatorname{Normal}[\boldsymbol{m}(t), \boldsymbol{C}(t)]
$$


where

$$
\boldsymbol{m}(t)=\boldsymbol{a}(t)+\boldsymbol{K}(t)[\boldsymbol{Y}(t)-\boldsymbol{f}(t)] \quad \boldsymbol{C}(t)=\boldsymbol{R}(t)-\boldsymbol{K}(t) \boldsymbol{Q}(t) \boldsymbol{K}^{\prime}(t)
$$

and $\boldsymbol{K}(t)=\boldsymbol{R}(t) \boldsymbol{F}_{d}^{\prime} \boldsymbol{Q}(t)^{-1}$ is the Kalman gain at time $t$.

The expected state at time $t=0$ for the abrupt $4 \times \mathrm{CO}_{2}$ experiment is given by $\boldsymbol{m}(0)=\left(2 F_{C}, 0,0,0\right)^{\prime}$, i.e., $4 \times \mathrm{CO}_{2}$ forcing from Equation 6 applied to an initial equilibrium state. The covariance $\boldsymbol{C}(0)$ of the state at time $t=0$ is taken to be the stationary marginal covariance of the EBM with only stochastic forcing Cummins et al. 2020, Appendix C].

\section{B Projections and standardised errors}

Likelihood theory tells us that the asymptotic distribution of the maximum likelihood estimator $\hat{\phi}$ of the true parameters $\phi$ is

$$
\hat{\phi} \sim \operatorname{Normal}\left[\phi, I(\phi)^{-1}\right]
$$

where $I(\phi)$ is the expected information matrix. The information matrix can be computed numerically as part of the optimisation procedure used to find $\hat{\theta}$.

Therefore, given $\hat{\phi}$ and equivalent $\mathrm{CO}_{2}$ forcings $X(t)$ for the historical and RCP4.5 experiments, we can sample the full projection uncertainty due to natural variability and parameter uncertainty for each fitted model as follows:

- Sample $\phi^{\star} \sim \operatorname{Normal}\left[\hat{\phi}, I(\hat{\phi})^{-1}\right]$;

- Let $\boldsymbol{\theta}^{\star}(0)=(0,0,0,0)^{\prime}$, i.e., pre-industrial equilibrium;

- For $t$ in $1, \ldots, T$

- Sample $\boldsymbol{\theta}^{\star}(t)$ from Equation 8, conditional on $\boldsymbol{\theta}^{\star}(t-1), X(t)$ and $\boldsymbol{\phi}^{\star}$;

- Sample $\boldsymbol{Y}^{\star}(t)$ from Equation 7, conditional on $\boldsymbol{\theta}^{\star}(t)$ and $\boldsymbol{\phi}^{\star}$;

By repeating the sampling procedure we can obtain as many samples $\boldsymbol{Y}^{\star}(1), \ldots, \boldsymbol{Y}^{\star}(T)$ as we wish, sampling the full extent of both the parameter uncertainty and natural variability.

The standardised prediction errors at each time $t$ are then defined as

$$
\frac{Y(t)-\mathrm{E}\left[Y^{\star}(t)\right]}{\sqrt{\operatorname{Var}\left[Y^{\star}(t)\right]}}
$$

where $Y(t)$ is the climate model output for the historical/RCP4.5 scenario at time $t$ and $\mathrm{E}\left[Y^{\star}(t)\right]$ and $\operatorname{Var}\left[Y^{\star}(t)\right]$ are estimated from the samples $Y^{\star}(t)$. 


\section{Extended state-space representation}

The state-space representation of the extended model given by Equations 914 conditioned on the shared discrepancy $\nu(t)$ is given by

$$
\boldsymbol{D}=\left[\begin{array}{ccc}
\gamma F_{C} & \gamma F_{V} & 0 \\
0 & 0 & 0 \\
0 & 0 & 0 \\
0 & 0 & 0 \\
0 & 0 & 1
\end{array}\right] \quad \boldsymbol{X}(t)=\left[\begin{array}{c}
X_{C}(t) \\
X_{V}(t) \\
\nu(t)
\end{array}\right]
$$

and

$$
\boldsymbol{G}=\left[\begin{array}{ccccc}
-\gamma & 0 & 0 & 0 & 0 \\
\frac{1}{C_{1}} & -\frac{k_{1}+k_{2}}{C_{1}} & \frac{k_{2}}{C_{1}} & 0 & \frac{1}{C_{1}} \\
0 & \frac{k_{2}}{C_{2}} & -\frac{k_{2}+\varepsilon k_{3}}{C_{2}} & \frac{\varepsilon k_{3}}{C_{2}} & 0 \\
0 & 0 & \frac{k_{3}}{C_{3}} & -\frac{k_{3}}{C_{3}} & 0 \\
0 & 0 & 0 & 0 & 0
\end{array}\right] \quad \boldsymbol{W}=\left[\begin{array}{ccccc}
\sigma_{F}^{2} & 0 & 0 & 0 & 0 \\
0 & \frac{\sigma_{T}^{2}}{C_{1}^{2}} & 0 & 0 & 0 \\
0 & 0 & 0 & 0 & 0 \\
0 & 0 & 0 & 0 & 0 \\
0 & 0 & 0 & 0 & \sigma_{\delta}^{2}
\end{array}\right] .
$$

The discretised version is

$$
\boldsymbol{F}_{d}=\left[\begin{array}{ccccc}
0 & 1 & 0 & 0 & 0 \\
1 & -k_{1} & (1-\varepsilon) k_{3} & -(1-\varepsilon) k_{3} & 1
\end{array}\right] \quad \quad \boldsymbol{V}_{d}=\left[\begin{array}{ll}
0 & 0 \\
0 & 0
\end{array}\right]
$$

and

$$
\boldsymbol{G}_{d}=e^{\boldsymbol{G}} \quad \boldsymbol{D}_{d}=\boldsymbol{G}^{-1}\left(\boldsymbol{G}_{d}-\boldsymbol{I}\right) \boldsymbol{D} \quad \boldsymbol{W}_{d}=\int_{s=0}^{1} e^{\boldsymbol{G} s} \boldsymbol{W} e^{\boldsymbol{G}^{\prime} s}
$$

\section{${ }_{553}$ References}

Abramowitz, G., and C. H. Bishop, Climate model dependence and the ensemble dependence transformation of CMIP projections, Journal of Climate, 28(6), 2332-2348, doi:10.1175/JCLI-D-14-00364.1, 2015

Annan, J. D., and J. C. Hargreaves, Reliability of the CMIP3 ensemble, Geophysical Research Letters, 37, L02,703, doi:10.1029/2009GL041994, 2010.

Annan, J. D., and J. C. Hargreaves, Understanding the CMIP3 multimodel ensemble, Journal of Climate, 24(16), 4529-4538, doi:10.1175/2011JCLI3873.1, 2011. 
Bhat, K. S., M. Haran, A. Terando, and K. Keller, Climate Projections Using Bayesian Model Averaging and Space-Time Dependence, Journal of Agricultural, Biological, and Environmental Statistics, 16(4), 606-628, doi:10.1007/sl3253-011-0069-3, 2011.

Brient, F., Reducing uncertainties in climate projections with emergent constraints: Concepts, examples and prospects, Advances in Atmospheric Sciences, 37, 1-15, doi:10.1007/s00376-019-9140-8, 2020.

Broecker, J., Probability Forecasts, in Forecast Verification: A Practicioner's Guide in Atmostpheric Science, edited by I. T. Joliffe and D. B. Stephenson, second ed., pp. 119-140, John Wiley \& Sons, Ltd., 2012.

Buser, C. M., H. R. Künsch, D. Lüthi, M. Wild, and C. Schär, Bayesian multi-model projection of climate: Bias assumptions and interannual variability, Climate Dynamics, 33(6), 849-868, doi:10. 1007/s00382-009-0588-6, 2009.

Caldwell, P. M., M. D. Zelinka, and S. A. Klein, Evaluating emergent constraints on equilibrium climate sensitivity, Journal of Climate, 31(10), 3921-3942, doi:10.1175/JCLI-D-17-0631.1, 2018.

Chandler, R. E., Exploiting strength, discounting weakness: combining information from multiple climate simulators, Philosophical Transactions of the Royal Society A: Mathematical, Physical and Engineering Sciences, 371, 20120,388, doi:10.1098/rsta.2012.0388, 2013.

Collins, M., Ensembles and probabilities: A new era in the prediction of climate change, Philosophical Transactions of the Royal Society A: Mathematical, Physical and Engineering Sciences, 365(1857), 1957-1970, doi:10.1098/rsta.2007.2068, 2007.

Collins, M., et al., Long-term Climate Change: Projections, Commitments and Irreversibility, in Climate Change 2013: The Physical Science Basis, edited by T. F. Stocker, D. Qin, G.-K. Plattner, M. M. B. Tignor, S. K. Allen, J. Boschung, A. Nauels, Y. Xia, V. Bex, and P. M. Midgley, Cambridge University Press, 2013

Cox, P. M., C. Huntingford, and M. S. Williamson, Emergent constraint on equilibrium climate sensitivity from global temperature variability, Nature, 553(7688), 319-322, doi:10.1038/nature25450, 2018.

Cummins, D. P., D. B. Stephenson, and P. A. Stott, Optimal Estimation of Stochastic Energy Balance Model Parameters, Journal of Climate, 2020.

Deser, C., A. S. Phillips, V. Bourdette, and H. Teng, Uncertainty in climate change projections: the role of internal variability, Climate Dynamics, 38, 527-546, doi:10.1007/s00382-010-0977-x, 2012.

Driscoll, S., A. Bozzo, L. J. Gray, A. Robock, and G. Stenchikov, Coupled Model Intercomparison Project 5 (CMIP5) simulations of climate following volcanic eruptions, Journal of Geophysical Research Atmospheres, 117(17), doi:10.1029/2012JD017607, 2012. 
Durbin, J., and S. J. Koopman, Time Series Analysis by State Space Methods, 2nd ed., 368 pp., Oxford University Press, 2012.

Fredriksen, H.-B., and M. Rypdal, Long-range persistence in global surface temperatures explained by linear multibox energy balance models, Journal of Climate, 30(18), 7157-7168, doi:10.1175/ JCLI-D-16-0877.1, 2017.

Furrer, R., S. R. Sain, D. W. Nychka, and G. A. Meehl, Multivariate Bayesian analysis of atmosphereocean general circulation models, Environmental and Ecological Statistics, 14(3), 249-266, doi:10. 1007/s10651-007-0018-z, 2007.

Gelman, A., and D. B. Rubin, Inference from Iterative Simulation Using Multiple Sequences, Statistical Science, 7(4), 457-511, doi:10.1214/ss/1177011136, 1992.

Geoffroy, O., D. Saint-Martin, D. J. L. Olivié, A. Voldoire, G. Bellon, and S. Tytéca, Transient Climate Response in a Two-Layer Energy-Balance Model. Part I: Analytical Solution and Parameter Calibration Using CMIP5 AOGCM Experiments, Journal of Climate, 26(6), 1841-1857, doi: 10.1175/JCLI-D-12-00195.1, 2013

Greene, A. M., L. Goddard, and U. Lall, Probabilistic multimodel regional temperature change projections, Journal of Climate, 19(17), 4326-4343, doi:10.1175/JCLI3864.1, 2006.

Gregory, J. M., W. J. Ingram, M. A. Palmer, G. S. Jones, P. A. Stott, R. B. Thorpe, J. A. Lowe, T. C. Johns, and K. D. Williams, A new method for diagnosing radiative forcing and climate sensitivity, Geophysical Research Letters, 31(3), 2-5, doi:10.1029/2003GL018747, 2004.

Hall, A. D., P. M. Cox, C. Huntingford, and S. A. Klein, Progressing emergent constraints on future climate change, Nature Climate Change, 9(4), 269-278, doi:10.1038/s41558-019-0436-6, 2019.

Hasselmann, K., Stochastic climate models Part I. Theory, Tellus, 28(6), 473-485, doi:10.3402/tellusa. v28i6.11316, 1976.

Held, I. M., M. Winton, K. Takahashi, T. L. Delworth, F. Zeng, and G. K. Vallis, Probing the fast and slow components of global warming by returning abruptly to preindustrial forcing, Journal of Climate, 23(9), 2418-2427, doi:10.1175/2009JCLI3466.1, 2010.

Huang, H., D. Hammerling, B. Li, and R. L. Smith, Combining interdependent climate model outputs in CMIP5: A spatial Bayesian approach, 2020.

IPCC, Global Warming of $1.5^{\circ} \mathrm{C}$. An IPCC Special Report on the impacts of global warming of $1.5^{\circ} \mathrm{C}$ above pre-industrial levels and related global greenhouse gas emission pathways, in the context of strengthening the global response to the threat of climate change, 2018. 
Jiménez-de-la Cuesta, D., and T. Mauritsen, Emergent constraints on Earth's transient and equilibrium response to doubled CO2 from post-1970s global warming, Nature Geoscience, 12(11), 902-905, doi: 10.1038/s41561-019-0463-y, 2019.

Jonko, A., N. M. Urban, and B. Nadiga, Towards Bayesian hierarchical inference of equilibrium climate sensitivity from a combination of CMIP5 climate models and observational data, Climatic Change, 149(2), 247-260, doi:10.1007/s10584-018-2232-0, 2018.

Knutti, R., G. Abramowitz, M. Collins, V. Eyring, P. J. Gleckler, B. Hewitson, and L. O. Mearns, Good Practice Guidance Paper on Assessing and Combining Multi Model Climate Projections, in Meeting report of the Intergovernmental Panel On Climate Change Expert Meeting on Assessing and Combining Multiple Model Climate Projections, edited by T. Stocker, Q. Dahe, G.-K. Plattner, M. Tignor, and P. Midgley, \{IPCC $\}$ Working Group $\{$ I $\}$ Technical Support Unit, 2010.

Knutti, R., J. Sedláček, B. M. Sanderson, R. Lorenz, E. M. Fischer, and V. Eyring, A climate model projection weighting scheme accounting for performance and interdependence, Geophysical Research Letters, 44(4), 1909-1918, doi:10.1002/2016GL072012, 2017.

Meehl, G. A., C. A. Senior, V. Eyring, G. Flato, J. F. Lamarque, R. J. Stouffer, K. E. Taylor, and M. Schlund, Context for interpreting equilibrium climate sensitivity and transient climate response from the CMIP6 Earth system models, Science Advances, 6(26), 1-11, doi:10.1126/sciadv.aba1981, 2020.

Meinshausen, M., et al., The RCP greenhouse gas concentrations and their extensions from 1765 to 2300, Climatic Change, 109(1), 213-241, doi:10.1007/s10584-011-0156-z, 2011.

Min, S. K., and A. Hense, A Bayesian approach to climate model evaluation and multi-model averaging with an application to global mean surface temperatures from IPCC AR4 coupled climate models, Geophysical Research Letters, 33(8), L08,708, doi:10.1029/2006GL025779, 2006.

Morice, C. P., J. J. Kennedy, N. A. Rayner, and P. D. Jones, Quantifying uncertainties in global and regional temperature change using an ensemble of observational estimates: The HadCRUT4 data set, Journal of Geophysical Research Atmospheres, 117(8), 1-22, doi:10.1029/2011JD017187, 2012.

Moss, R. H., et al., The next generation of scenarios for climate change research and assessment, Nature, 463(7282), 747-756, doi:10.1038/nature08823, 2010.

Nijsse, F. J. M. M., P. M. Cox, and M. S. Williamson, An emergent constraint on Transient Climate Response from simulated historical warming in CMIP6 models, Earth System Dynamics Discussions, pp. 1-14, doi:10.5194/esd-2019-86, 2020. 
Palmer, T. N., R. Buizza, R. Hagedorn, A. Lawrence, M. Leutbecher, and L. A. Smith, Ensemble prediction: a pedagogical perspective, ECMWF Newsletter, 106, 10-17, doi:10.21957/ab129056ew, 2006.

Räisänen, J., and T. N. Palmer, A probability and decision-model analysis of a multimodel ensemble of climate change simulations, Journal of Climate, 14(15), 3212-3226, doi:10.1175/1520-0442(2001) 014〈3212:APADMA $\rangle 2.0 . C O ; 2,2001$.

Rougier, J. C., M. Goldstein, and L. House, Second-Order Exchangeability Analysis for Multimodel Ensembles, Journal of the American Statistical Association, 108(503), 852-863, doi:10.1080/01621459. 2013.802963, 2013.

Sanderson, B. M., M. Wehner, and R. Knutti, Skill and independence weighting for multi-model assessments, Geoscientific Model Development, 10(6), 2379-2395, doi:10.5194/gmd-10-2379-2017, 2017.

Sansom, P. G., D. B. Stephenson, C. A. T. Ferro, G. Zappa, and L. C. Shaffrey, Simple uncertainty frameworks for selecting weighting schemes and interpreting multimodel ensemble climate change experiments, Journal of Climate, 26, 4017-4037, doi:10.1175/JCLI-D-12-00462.1, 2013.

Sansom, P. G., D. B. Stephenson, and T. J. Bracegirdle, On constraining projections of future climate using observations and simulations from multiple climate models, Journal of the American Statistical Association, p. In review, 2020.

Sato, M., J. E. Hansen, M. P. McCormick, and J. B. Pollack, Stratospheric Aerosol Optical Depths, 1850-1990, 98, 22,987-22,994, doi:10.1029/93JD02553, 1993.

Sherwood, S. C., et al., An Assessment of Earth's Climate Sensitivity Using Multiple Lines of Evidence, Reviews of Geophysics, 58(4), 1-92, doi:10.1029/2019rg000678, 2020.

Shiogama, H., S. Emori, N. Hanasaki, M. Abe, Y. Masutomi, K. Takahashi, and T. Nozawa, Observational constraints indicate risk of drying in the Amazon basin., Nature communications, 2, 253, doi:10.1038/ncomms1252, 2011.

Smith, L. A., What might we learn from climate forecasts?, Proceedings of the National Academy of Sciences of the United States of America, 99 (SUPPL. 1), 2487-2492, doi:10.1073/pnas.012580599, 2002.

Smith, R. L., C. Tebaldi, D. W. Nychka, and L. O. Mearns, Bayesian Modeling of Uncertainty in Ensembles of Climate Models, Journal of the American Statistical Association, 104(485), 97-116, doi:10.1198/jasa.2009.0007, 2009. 
Stainforth, D. A., M. R. Allen, E. R. Tredger, and L. A. Smith, Confidence, uncertainty and decisionsupport relevance in climate predictions, Philosophical Transactions of the Royal Society A, 365, 21452161, doi:10.1098/rsta.2007.2074, 2007.

Stephenson, D. B., M. Collins, J. C. Rougier, and R. E. Chandler, Statistical problems in the probabilistic prediction of climate change, Environmetrics, 23(5), 364-372, doi:10.1002/env.2153, 2012.

Strobach, E., and G. Bel, Learning algorithms allow for improved reliability and accuracy of global mean surface temperature projections, Nature Communications, 11(1), 1-7, doi:10.1038/ s41467-020-14342-9, 2020.

Sutton, R. T., E. Suckling, and E. Hawkins, What does global mean temperature tell us about local climate?, Philosophical Transactions of the Royal Society A: Mathematical, Physical and Engineering Sciences, 373(2054), doi:10.1098/rsta.2014.0426, 2015.

Tebaldi, C., and R. Knutti, The use of the multi-model ensemble in probabilistic climate projections, Philosophical Transactions of the Royal Society A: Mathematical, Physical and Engineering Sciences, 365 (1857), 2053-2075, doi:10.1098/rsta.2007.2076, 2007.

Tebaldi, C., and B. Sansó, Joint projections of temperature and precipitation change from multiple climate models: A hierarchical Bayesian approach, Journal of the Royal Statistical Society: Series A (Statistics in Society), 172(1), 83-106, doi:10.1111/j.1467-985X.2008.00545.x, 2009.

Tebaldi, C., R. L. Smith, D. W. Nychka, and L. O. Mearns, Quantifying uncertainty in projections of regional climate change: A Bayesian approach to the analysis of multimodel ensembles, Journal of Climate, 18(10), 1524-1540, doi:10.1175/JCLI3363.1, 2005.

Tokarska, K. B., M. B. Stolpe, S. Sippel, E. M. Fischer, C. J. Smith, F. Lehner, and R. Knutti, Past warming trend constrains future warming in CMIP6 models, Science Advances, 6(12), 1-14, doi: 10.1126/sciadv.aaz9549, 2020

Vihola, M., Robust adaptive Metropolis algorithm with coerced acceptance rate, Statistics and Computing, 22(5), 997-1008, doi:10.1007/s11222-011-9269-5, 2012.

Watterson, I. G., and P. H. Whetton, Distributions of decadal means of temperature and precipitation change under global warming, Journal of Geophysical Research: Atmospheres, 116(7), 1-13, doi: 10.1029/2010JD014502, 2011.

Weigel, A. P., R. Knutti, M. A. Liniger, and C. Appenzeller, Risks of model weighting in multimodel climate projections, Journal of Climate, 23(15), 4175-4191, doi:10.1175/2010JCLI3594.1, 2010. 
West, M., and P. J. Harrison, Bayesian Forecasting and Dynamic Models, 2nd ed., 682 pp., SpringerVerlag New York, 1997.

Young, P. C., C. N. Ng, K. Lane, and D. Parker, Recursive Forecasting, Smoothing and Seasonal Adjustment of Nonstationary Environmental Data, Journal of Forecasting, 10(1-2), 57-89, doi: 10.1002/for.3980100105, 1991. 

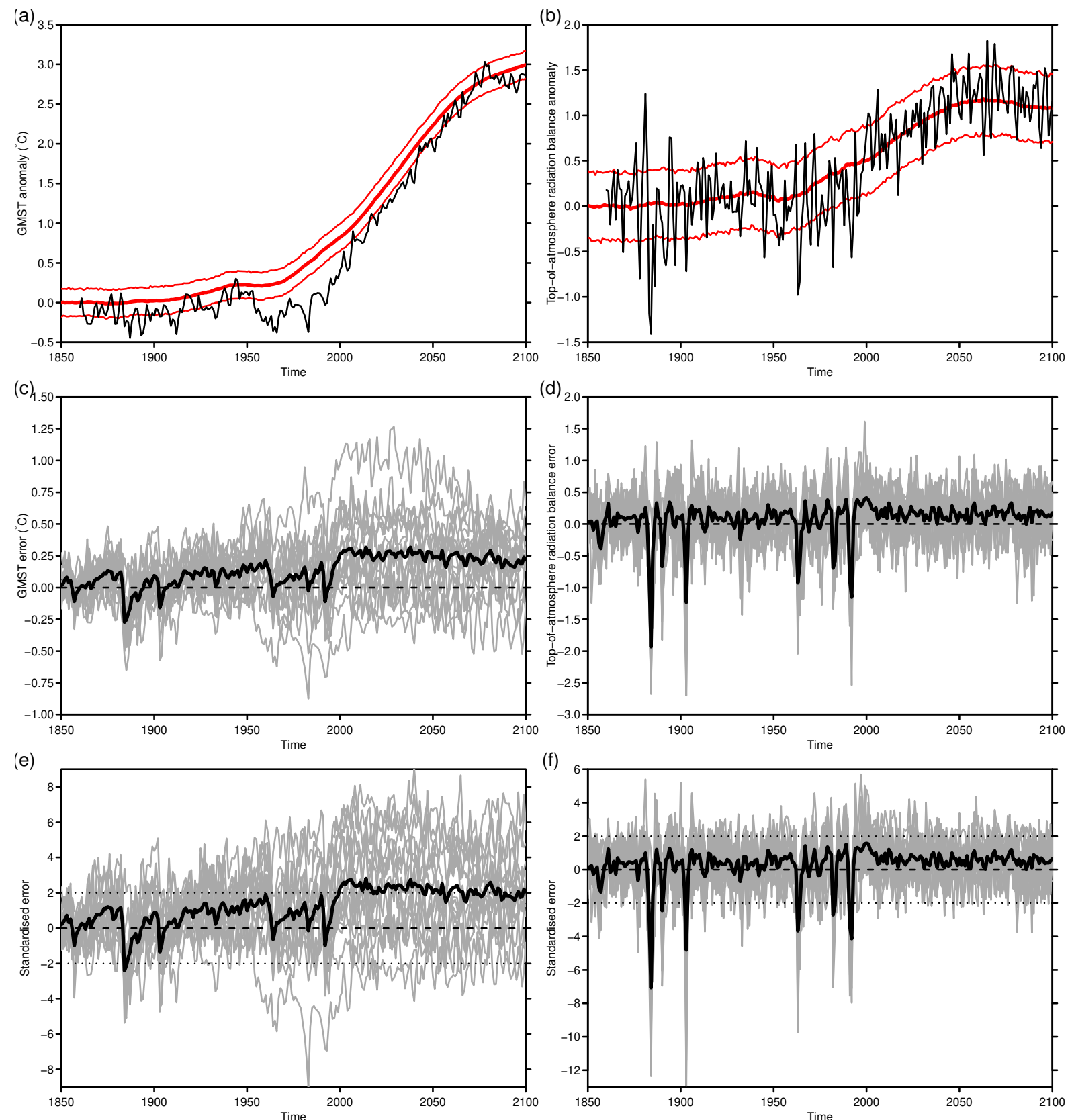

Figure 2: EBM projection reliability. (a) global mean surface temperature, and (b) top-of-atmosphere radiation balance in the CanESM2 model under the RCP4.5 scenario. Black lines are the model simulations, thick red lines are the posterior predictive means based on fitting the EBM to the abrupt4xCO2 scenario, and thin red lines are a marginal $90 \%$ credible interval. (c) and (d) Standardised prediction errors for global mean surface temperature, and top-of-atmosphere radiation balance respectively under the RCP4.5 scenario based on fitting to the abrupt4xCO2 scenario. Thin grey lines represent standardised errors from individual CMIP5 models. The thick black line is the ensemble mean. 


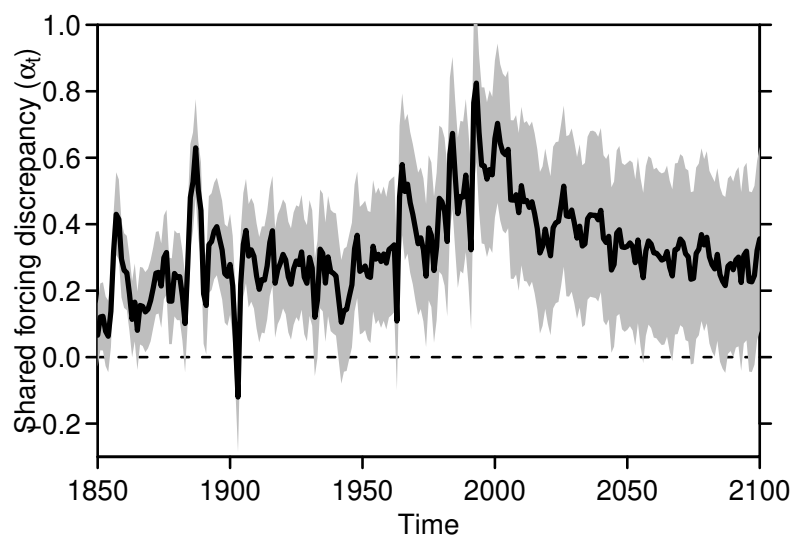

Figure 3: The shared forcing discrepancy. The posterior mean (solid line) and marginal $90 \%$ credible intervals (shading) for the shared forcing discrepancy $\alpha_{t}$ under the RCP4.5 forcing scenario. 

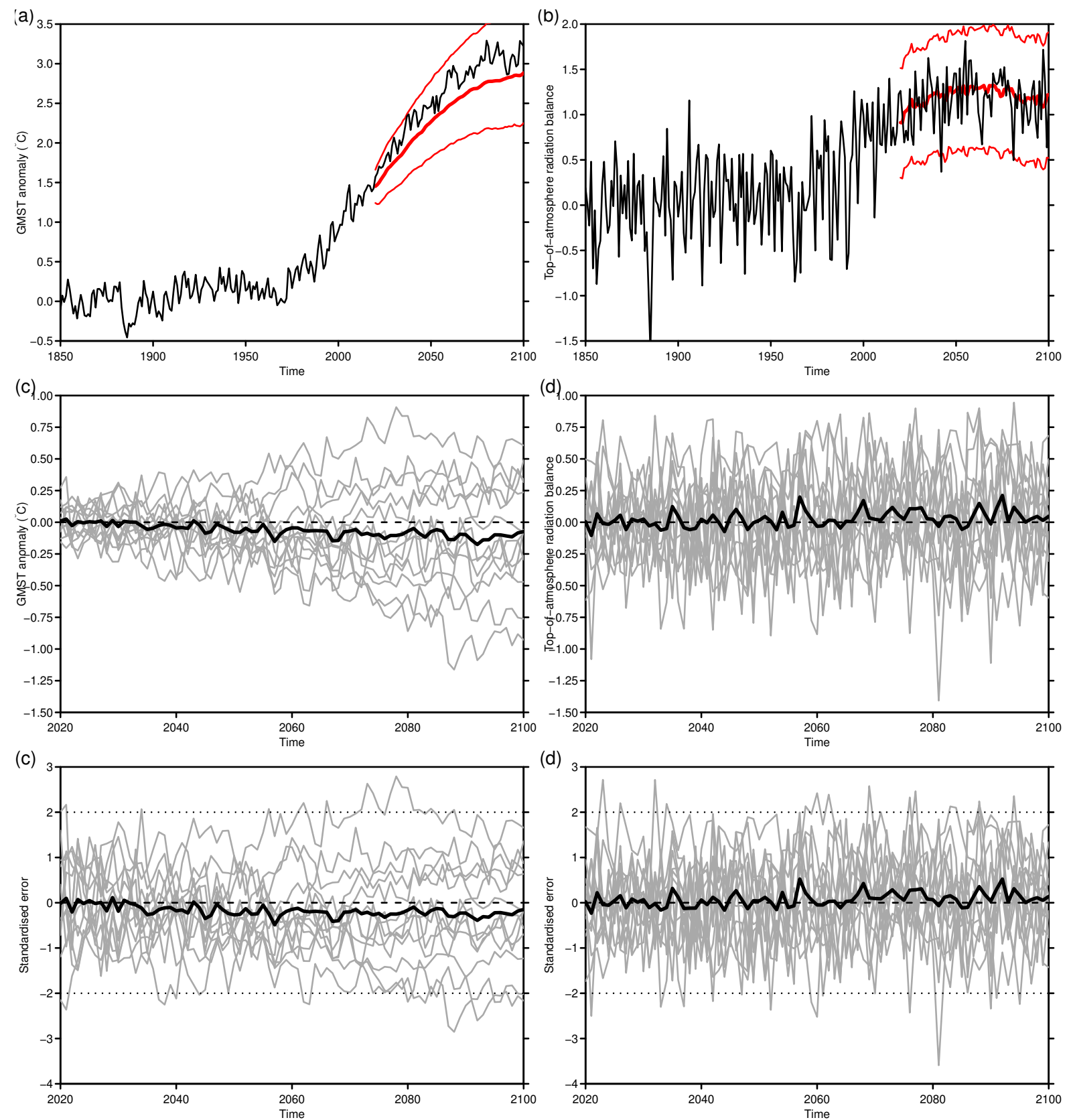

Figure 4: Cross validation. (a) and (b) global mean surface temperature and top-of-atmosphere radiation balance respectively in the CanESM2 model under the RCP4.5 scenario. Black lines are the model simulations, thick red lines are the posterior predictive means based on the model including both individual and shared forcing discrepancies, and thin red lines are a marginal $90 \%$ credible interval. (c) and (d) Standardised prediction errors for global mean surface temperature and top-of-atmosphere radiation balance respectively under the RCP4.5 scenario including both individual and shared forcing discrepancies. Thin grey lines represent standardised errors from individual CMIP5 models. The thick black line is the ensemble mean. 


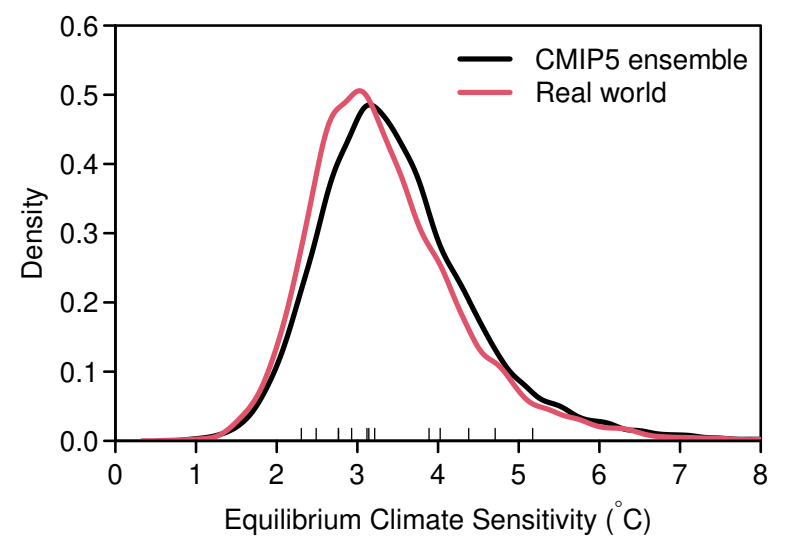

Figure 5: Equilibrium climate sensitivity. The posterior density of the equilibrium climate sensitivity for the real-world (red) and the CMIP5 ensemble (black).

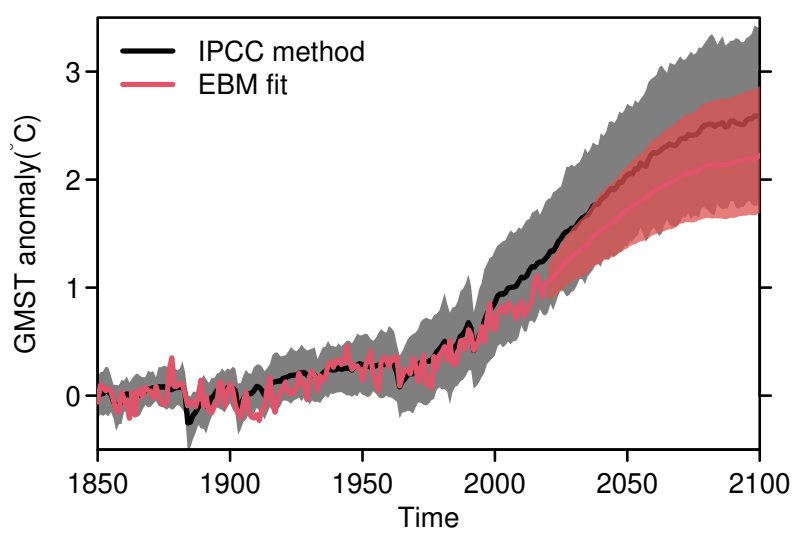

Figure 6: Global mean surface temperature anomaly projections. Posterior predictive distribution for GMST change under the RCP4.5 forcing scenario compared to the early industrial period (1850-1900). Thick lines indicate the IPCC mean projection (black), the observations (1850-2019) and posterior mean projection (2020-2100) by our EBM fitting method (red). Grey shading indicates the $90 \%$ interval based on the IPCC method. Red shading indicates a $90 \%$ credible interval based on our EBM method.
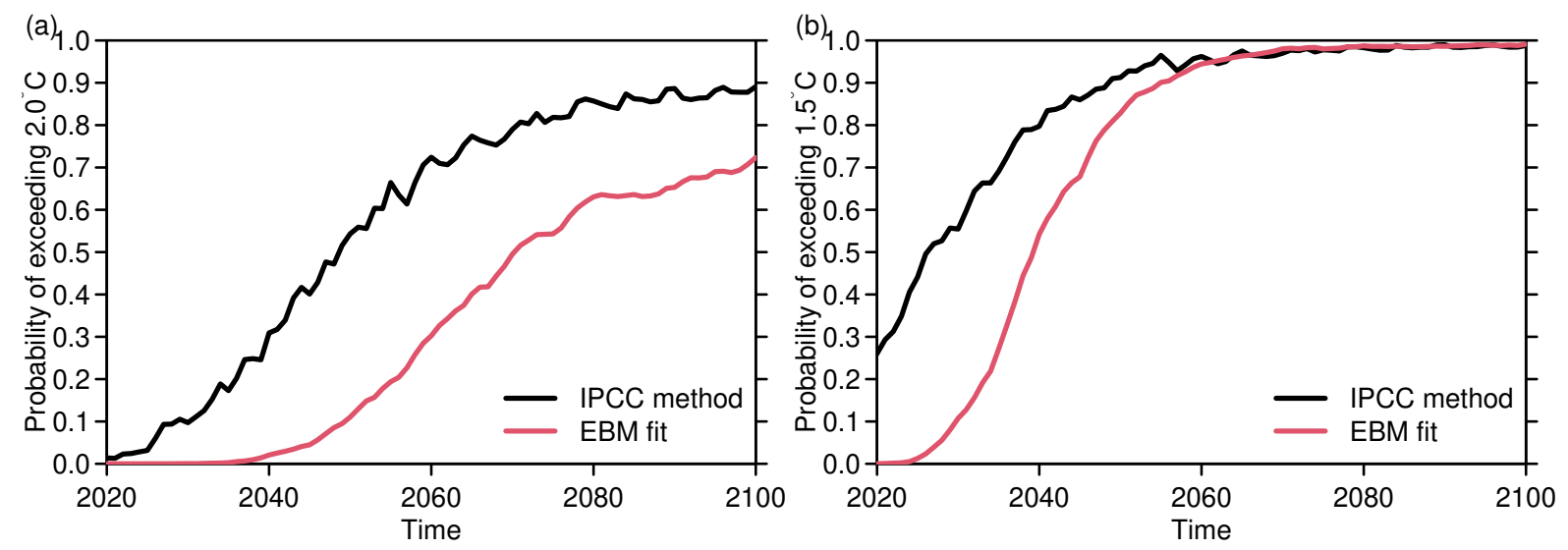

Figure 7: Probability of meeting the Paris agreement. (a) The probability of climate change exceeding $2.0^{\circ} \mathrm{C}$ under the RCP4.5 forcing scenario compared to the early industrial period (1850-1900), and (b) the probability of climate change exceeding $1.5^{\circ} \mathrm{C}$ under the RCP4.5 forcing scenario compared to the early industrial period (1850-1900). 


\section{Supplementary Files}

This is a list of supplementary files associated with this preprint. Click to download.

- supplementarymaterial.pdf 